\title{
Fortaleciendo el proceso de planificación didáctica de las educadoras del nivel inicial
}

\author{
Fabiola Gema Alexandra Genet Narváez \\ Universidad Nacional Autónoma de Nicaragua, Managua UNAN-Managua \\ fgenet010585@yahoo.es
}

Recibido: noviembre 2016 Aprobado: diciembre 2016

\section{RESUMEN}

En el presente artículo, se aborda: el Fortalecimiento de la planificación didáctica de las experiencias de aprendizaje de los niños de 0-3 años en el Centro Educativo San Martín de Porres, del municipio de Ciudad Sandino, en el período de abril-noviembre del año 2015. El interés por indagar sobre el tema, surgió por medio de las visitas realizadas al centro e interacciones con los niños, maestras y directora general del centro, que permitió visualizar dificultades en el proceso de planificación didáctica y aplicación del currículo de la niñez de 0-3 años. El enfoque del estudio, es cualitativo en la modalidad de investigación acción, descriptiva y transversal. Se seleccionó a seis docentes, directora y técnico del MIFAM. Al realizar el diagnóstico, los principales hallazgos, fueron: Que las docentes requieren de capacitación en planificación didáctica, ya que presentan debilidades en la selección de competencias, aprendizajes esperados y evidencias de aprendizaje, aplicación del programa de atención educativa a la niñez de 0-3 años, poca aplicación de estrategias oportunas, poca motivación y promoción por las autoridades para la elaboración del plan diario. De acuerdo a estos resultados se ejecutaron cuatro planes de acción con las docentes, obteniendo resultados significativos, entre ellos: mayor interés al realizar su planificación, estética del plan, mejor redacción. Finalmente se brindan recomendaciones pertinentes al Técnico del Mi Familia (MIFAM), Directora y Docentes del Centro educativo, tales como: brindar acompañamiento pedagógico a las educadoras, ofrecer material didáctico y complementario para la planificación del ciclo 1 de educación inicial y dar seguimiento a esta investigación.

Palabras Claves: Planificación Didáctica, Estrategias Didácticas, Educación Inicial, Capacitación.

\section{ABSTRACT}

This article discusses the Enrichment of lesson planning base don't helearning experience of 0-3 year-old children at the Centro Educativo San Martín de Porres in the Municipality of Ciudad Sandino from Aprilto November 2015. The interest of delving in to this issue comes as a result of observation visits, interaction with children, teachers and the principal that enabledusto encounter difficulties in the lesson planning process and in curriculum management for 0-3 year-old children. The approachusedis a qualitative, descriptive, and cross-sectionalaction research. Sixte achers weres elected, the principal and a representative of the MIFAM (Administration for Children and Families). Afterthediagnostic test wasrun, it was found that the teachers need training onlessonplanningsincetheylacktheabilitytochoosetheappropriatelearningcompetences, expected learning out comes and learning achievements. The yalsohaveweakness in the management of the school curriculum sed with 0-3 year-old children, little use of appropriate strategies, motivation and the promotion of lesson planning by the authorities. From these results four action plans were executed and meaningfuloutcomesobtainedsuch as a greater interest in the development of the lesson plan, its organization and wording. Finally, relevantre commendations are giventothe MIFAMrepresentative, principal and teachers through pedagogical supportto educators and extra teaching material supply forthel esson planning in early educational ong with a follow-up to this research.

Key words: Lesson Planning, Teaching Strategies, Early Education, Training 


\section{INTRODUCCIÓN}

Las autoridades de nuestro país, han reconocido el valor de la educación inicial, a través de políticas y programas a favor de la primera infancia. Así mismo las entidades responsables de la educación de nuestro país han desarrollado diversas estrategias para el mejoramiento de la calidad de la educación, tomando en cuenta como prioridad la educación de los más pequeños.

La investigación ha sido desarrollada con las educadoras del ciclo 1 de educación inicial del Centro Educativo San Martín de Porres, del municipio de Ciudad Sandino, quienes expresaron la necesidad de ser orientadas para utilizar el programa de atención educativa a la niñez de 0-3 años, un Programa relativamente nuevo. La mayoría de ellas no han sido formadas profesionalmente para atender educativamente a niños de 0-3 años, sin embargo cuentan con varios años de experiencia en esos niveles.

En visitas y observaciones realizadas se detectó que planificaban y desarrollaban solamente una estrategia metodológica para cada día de la semana. Esta situación era evidente, ya que la mayor parte del tiempo las educadoras brindaban atención de cuido a los niños y niñas, con pocas intervenciones pedagógicas. En cuanto a la planificación didáctica que realizan las educadoras se constató que la única forma de planificación es semanal.

El tema de investigación es novedoso y aún no se han realizado estudios sobre el mismo, sin embargo, se encontró una investigación que tiene relación con el tema de estudio: Situación actual del currículo 0-3 años en Nicaragua, en la ciudad de Managua, realizado por Aguilera, M., y Aguirre, M. (II semestre del año 2012). Dicha investigación, dirigió sus recomendaciones a las autoridades correspondientes, solicitando que finalizaran lo que hoy es la versión del Programa de Atención Educativa a la niñez de 0-3 años, ya que los Preescolares y Centros de Desarrollo Infantil, realizaban la planificación, pero de manera desorganizada, cada institución lo implementaba a su modo, y no utilizaban los mismos programas ni guías metodológicas.

Reyes y Uriarte (2012), realizaron una valoración sobre el desarrollo del 1er nivel del diplomado de atención a la primera infancia "Amor por los más chiquitos de 0-6 años", los participantes del diplomado, concluyen con una valoración positiva, ya que se capacitaron a las maestras y educadoras del nivel preescolar formal y centros comunitarios, con el propósito de actualizar sus conocimientos teóricos y transformar su práctica educativa con base a los nuevos enfoques de derecho y visión inclusiva en la educación inicial. 
La Educación Inicial actualmente se concibe como un derecho que tienen los niños y las niñas, desde que nacen hasta que cumplen seis años, en busca de promover su desarrollo integral, partiendo del reconocimiento de sus particularidades e intereses, del respeto hacia sus diferentes ritmos de desarrollo, de la importancia de explorar, jugar, crear, acercarse a la literatura, y del valor que tiene el compartir y aprender de adultos que los respetan, aman y protegen.

Según la Política de Primera Infancia, 2011, la primera infancia es el período fundamental de la vida desde el embarazo hasta los seis años de edad, cada niño es un ser integral, en su totalidad humana, física, cognitiva, afectiva, social y espiritual. Cada niño y niña en estas edades debe tener la oportunidad de contar con un ambiente estimulante protector, seguro, donde viva y se desarrolle saludable, emocionalmente seguro, socialmente cooperativo, solidario y con capacidad de aprender.

Todo ello, requiere planificar con intencionalidad todos los detalles, lejos de la improvisación, hasta aquellos puramente externos, es decir la ambientación del aula, asientos, mobiliario, material creado y utilizado, rincones, para configurar un entorno que condiciona la vida y la conducta de los sujetos que en ella interactúa. (Gervilla, 2006).

Manhey (2012), plantea que la planificación constituye el sello de la acción del docente, la que lo dota de profesionalismo a la actividad en la educación dirigida a los niños y niñas pequeños y propicia dar respuesta al reto de la educación infantil. La planificación es muy importante para el docente y los niños bajo su responsabilidad, ya que a través de esta el docente evita la improvisación, facilita su intervención y guía su práctica educativa en el aprendizaje y desarrollo del niño.

Tomando en cuenta estos planteamientos y la necesidad de las educadoras, resulta de mucha importancia compartir los hallazgos de esta investigación de manera que pueda ser analizada por otros investigadores interesados en la temática y por las instancias del Ministerio de la Familia quienes brindan asesoramiento a las educadoras de los centros que atienden a niños de 0-3 años.

La investigación refleja la necesidad de las educadoras de obtener nuevas oportunidades de capacitación que permitan mayor claridad de los procedimientos de aplicación del currículo 0-3 y la forma de ejecución de la metodología expresada en dicho instrumento pedagógico.

Por tal razón, se aborda la realidad educativa de las docentes que atienden a los niños y niñas de 0-3 años, del centro con el fin de comprender la planificación que realizan, concretar las dificultades encontradas y aportar mejoras, que benefician el proceso de enseñanza y aprendizaje, tomando en cuenta la 
importancia del tema ya que en esta etapa tan crucial se sientan las bases para el desarrollo posterior de los niños.

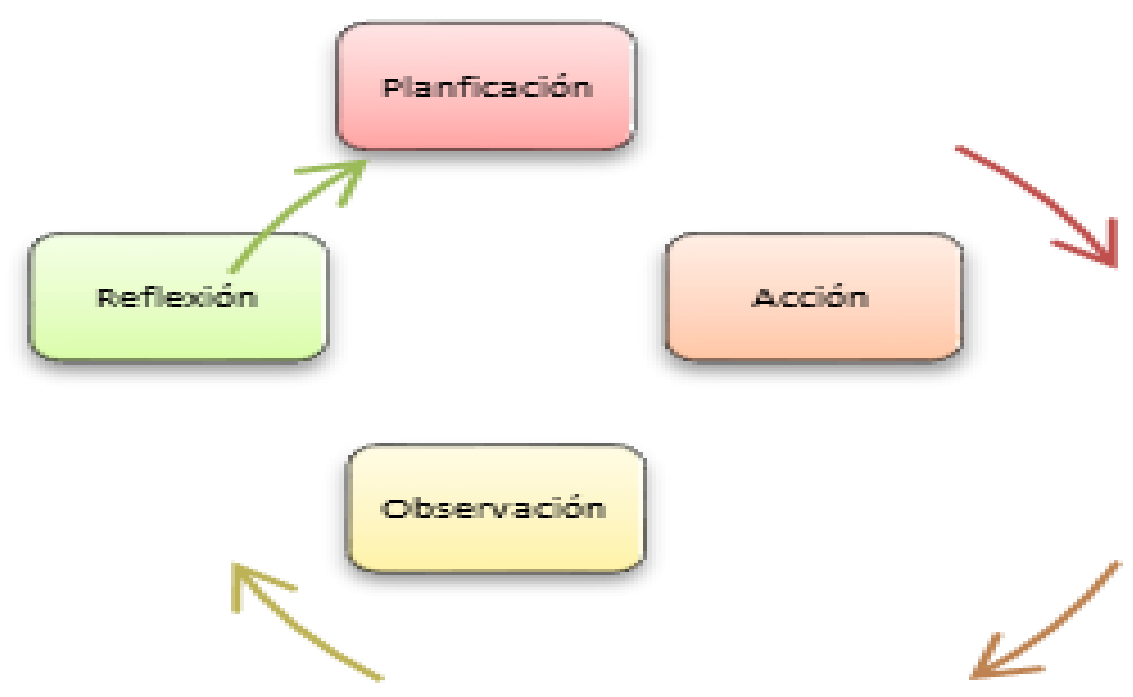

Figura 1. Metodología del Enfoque Investigación-acción

\section{MATERIAL Y MÉTODO}

Este estudio, tiene un enfoque de Investigación- Acción, que según Antonio Latorre (2005) es aquel que permite al investigador incidir propositivamente en la situación detectada, de tal modo que se aporte a resolver el problema. Este autor propone una metodología para este enfoque y sugiere fases o etapas para llevarlo a cabo:

Cada una de las etapas posee características específicas, y todas se desarrollaron en este estudio. Para obtener la información requerida en la elaboración de este trabajo, se seleccionó a las personas involucradas en los procesos de planificación y atención a los niños del ciclo 1 de educación inicial del centro.

Entre ellos, las 6 educadoras de los niveles de Lactantes ( 0 meses-1año), Infantes A (1 a 2 años) e Infantes B (2-3 años), por ser quienes elaboran y ejecutan la planificación didáctica de cada sala. También se solicitó información a la directora, ya que ella dirige, orienta y organiza el trabajo pedagógico que realizan las educadoras del ciclo 1, y al Técnico del Ministerio de la Familia, encargado del acompañamiento y asesoramiento de las educadoras de los centros que brindan atención a niños menores de tres años, del municipio de Ciudad Sandino. 
El estudio fue realizado en el marco de la atención a la primera infancia, en el Centro Educativo San Martín de Porres, ubicado en la segunda etapa del barrio Nueva Vida, en el Municipio de Ciudad Sandino, y sus barrios aledaños son: Carolina Calero y la Comunidad la Trinidad. También se visitó la delegación del Ministerio de la Familia de Ciudad Sandino para entrevistar al técnico que asesora a las educadoras.

Definidos los propósitos de la investigación se diseñaron los instrumentos de recolección de datos, entre ellos: entrevista, guías de observación de clase, grupo focal y análisis documental: revisión de planes y programas, por medio de los cuales se delimitó el problema.

Las entrevistas se realizaron en el siguiente orden: el día miércoles 15 de abril del año 2015, a las educadoras. El día 29 de abril se entrevistó a la directora del centro y al técnico del MIFAM.

Las observaciones realizadas a las educadoras con el fin de constatar la aplicación de las estrategias planteadas en el plan semanal se realizaron los días 22 y 29 de abril en este último se conformó un grupo focal, para enriquecer la información obtenida a través de las entrevistas, en el cual participaron tres de las seis educadoras.

El análisis documental se hizo en siete visitas al centro, solicitando el plan semanal a las educadoras, se tomaban fotografías de los mismos y se comparaban con el programa de atención educativa a la niñez de 0-3 años del Ministerio de la Familia.

Posterior al análisis de la información se aplicaron 4 planes de acción, por medio de talleres de capacitación realizados aproximadamente en dos horas, en el momento que los niños descansaban, llevados a cabo los días 13 y 20 de mayo, y 3 y 9 de junio del 2015.

Como parte del enfoque utilizado, se analizaron los resultados obtenidos por medio de la ejecución de los planes de acción, y se dio seguimiento al mismo. El día miércoles 25 de noviembre del presente año, se visitó el centro con el fin de observar la situación posterior a las intervenciones realizadas, solicitando a las educadoras facilitar los planes de clase para observar las características de estos, constatando si las acciones realizadas funcionaron y tuvieron efecto en la práctica educativa de las educadoras.

Para tal fin se hicieron observaciones y tomaron fotografías para registrar evidencias de las mejoras encontradas en los planes semanales de los tres niveles: Lactantes Infantes A y B, 
relacionándolos con los planes que presentaron las educadoras en el mes de marzo, momento en que dio inicio la investigación.

Los criterios regulativos utilizados en el estudio fueron: La triangulación y la Confirmabilidad.

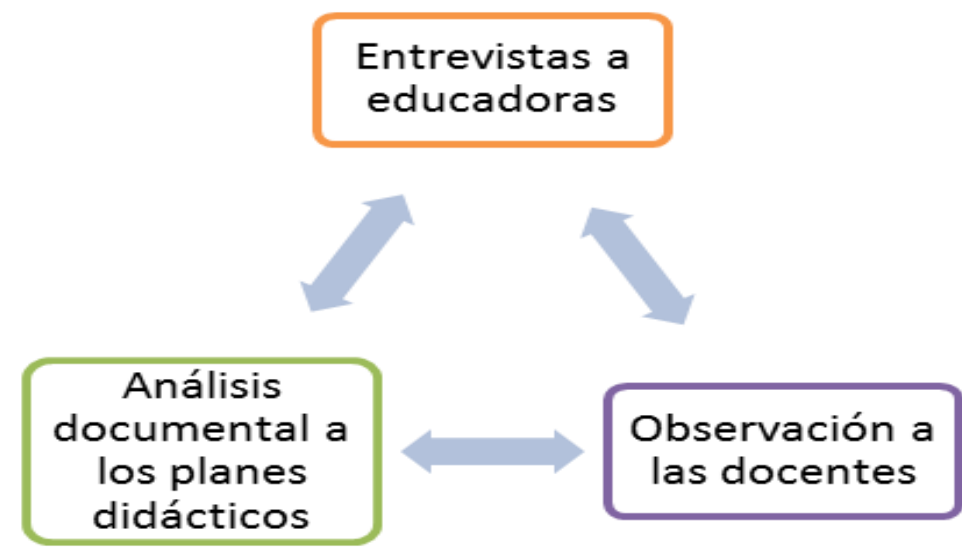

Figura 2. Fuente de información para la triangulación

Para dar cumplimiento al criterio regulativo de Confirmabilidad, se coordinó una reunión con las educadoras, directora y tutora en la que se dio a conocer los resultados del estudio y se destacaron los logros obtenidos producto de los planes de acción realizados. Así mismo se entregó al centro educativo un documento en físico del informe de todo lo actuado en el estudio.

Las técnicas para analizar la información fueron a través del diseño de matrices de 3 y 4 entradas para registrar la información proveniente de las entrevistas y guías de observación a los procesos educativos en las salas del 1 ciclo de educación inicial.

Para ordenar la información obtenida de diseñaron matrices con los resultados de las entrevistas realizadas a las seis docentes de manera que se apreciaran las seis respuestas para cada una de las preguntas realizadas, haciendo finalmente un consolidado por frecuencias de respuesta.

Además se organizó a través de esta técnica la información obtenida por medio de las entrevistas realizadas a la directora y técnico del ministerio de la familia, ya que ambas perseguían un mismo objetivo, de igual manera se consolidaron las respuestas de ambas partes.

Para organizar la información obtenida a través del grupo focal, primeramente se grabó la información utilizando un celular, y posteriormente se extrajo la información tomando en cuenta las preguntas redactadas para tal fin. Las guías de observación se complementaron con las anotaciones del 
diario de campo, para seleccionar la información relevante, relacionada al diagnóstico y ejecuciones de planes de acción para luego redactarla en el informe.

\section{RESULTADOS}

En esta investigación se plantearon objetivos en dos momentos, uno para realizar el diagnóstico y posteriormente para llevar a cabo los planes de acción que surgieron de las necesidades encontradas.

La etapa de diagnóstico tenía como objetivo general, valorar las necesidades metodológicas del proceso de planificación didáctica de las docentes del ciclo 1 de educación inicial. Los principales hallazgos fueron que las docentes tenían interés en ser capacitadas y experiencia en educación inicial.

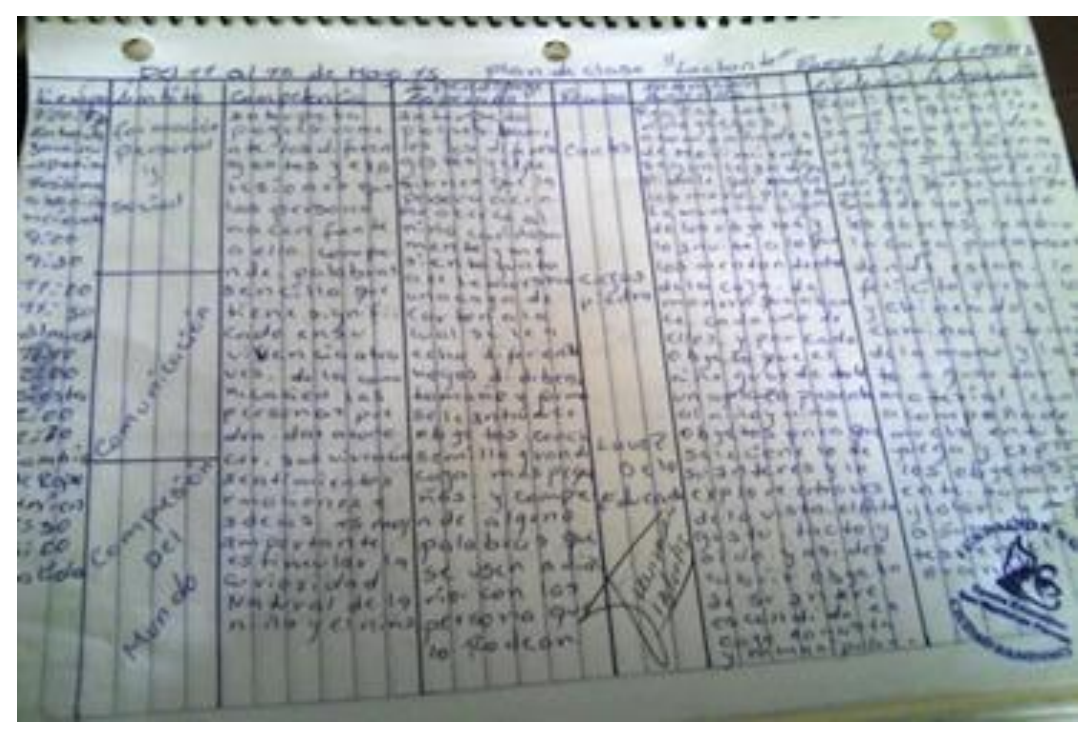

Figura 3. Planificación didáctica

Dentro de las dificultades se encontraron: Falta de capacitación, poco manejo y conocimiento de la aplicación del programa de atención educativa a la niñez de 0-3 años y la planificación didáctica carecía de variedad y cantidad de estrategias, para desarrollar con los niños en el transcurso de una semana. Sumado a esto algunas de las educadoras aplican estrategias con el fin a adelantar procesos que según ellas facilitan el aprendizaje en el primer nivel de preescolar, obviando otras que sí son pertinentes para esa etapa de la vida.

Las necesidades de capacitación estaban dirigidas a orientar a las educadoras en la forma adecuada de utilizar el programa de atención educativa a la niñez de 0-3 años para su planificación, y explicar algunos términos relacionados a los elementos básicos del currículo 0-3. 
Posteriormente se formuló la siguiente Hipótesis de Acción: “La capacitación teórico-práctica, fortalecerá el proceso de planificación didáctica de las docentes de primer ciclo de educación inicial del Centro Educativo San Martín de Porres”, y se procedió a plantear y ejecutar los cuatro planes de acción.

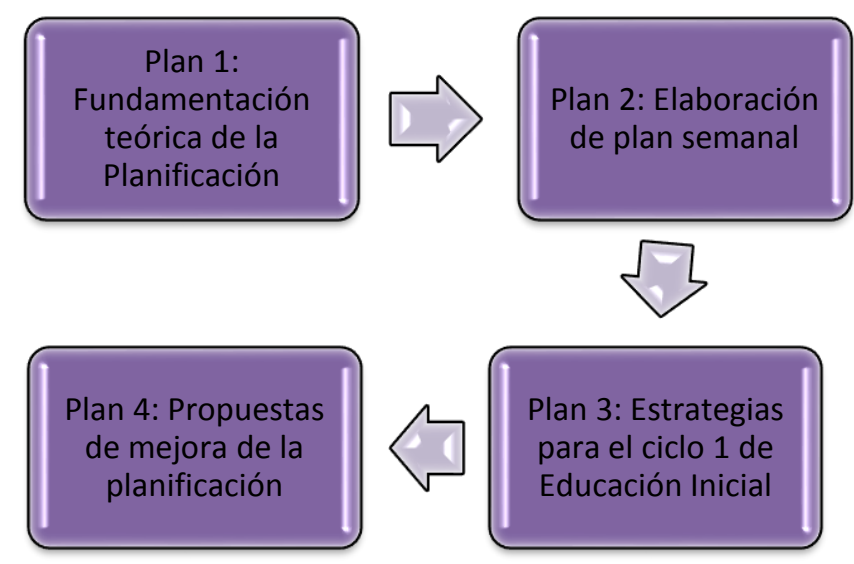

Figura 4. Planes de acción ejecutados

Los resultados obtenidos fueron los siguientes: En los planes de acción las educadoras expresaron interés por aprender nuevas formas de planificación, no obstante ellas mismas hacían la reflexión en cuanto a la variedad de orientaciones que reciben, lo cual les causa confusión y cierta incertidumbre, al no tener la capacidad de decidir y tomar acciones que mejoren su práctica educativa.

Las educadoras demostraron tener experiencias que resultó provechoso para la comprensión de nuevos conceptos y propuestas de otras alternativas de planificación.

En el proceso de seguimiento, al comparar los planes iniciales con los planes realizados posterior a la investigación, se observó lo siguiente: Mejor presentación y estética, incorporación de recursos y objetos concretos con los que el niño interactuó mediante las estrategias que se aplicaron, mayor dedicación en la realización del plan de clase, mejor claridad entre los objetivos y la incorporación de estrategias adecuadas para los niños de estas edades, tales como: el cuento, diferentes juegos, observación de láminas, manipulación de objetos, expresiones gestuales y corporales, interacción con el medio, dedican tiempo a la estética y presentación del plan, su plan ya no es de una página, ahora utilizan una página para cada ámbito en el que se aprecia mayor cantidad de estrategias propuestas, y principalmente se observa mayor aprovechamiento del tiempo, describiendo las actividades y sus intenciones pedagógicas. 
En la entrevista realizada a la Directora del Centro Educativo, expresó que las educadoras estaban satisfechas, porque se les ayudó y se enseñó a planificar, a través del acompañamiento brindado y aseveró lo siguiente: "han mejorado sus planes, ahora da gusto leerlos", han desarrollado un poco la creatividad, les hacen dibujos, los pintan y la información es más clara".

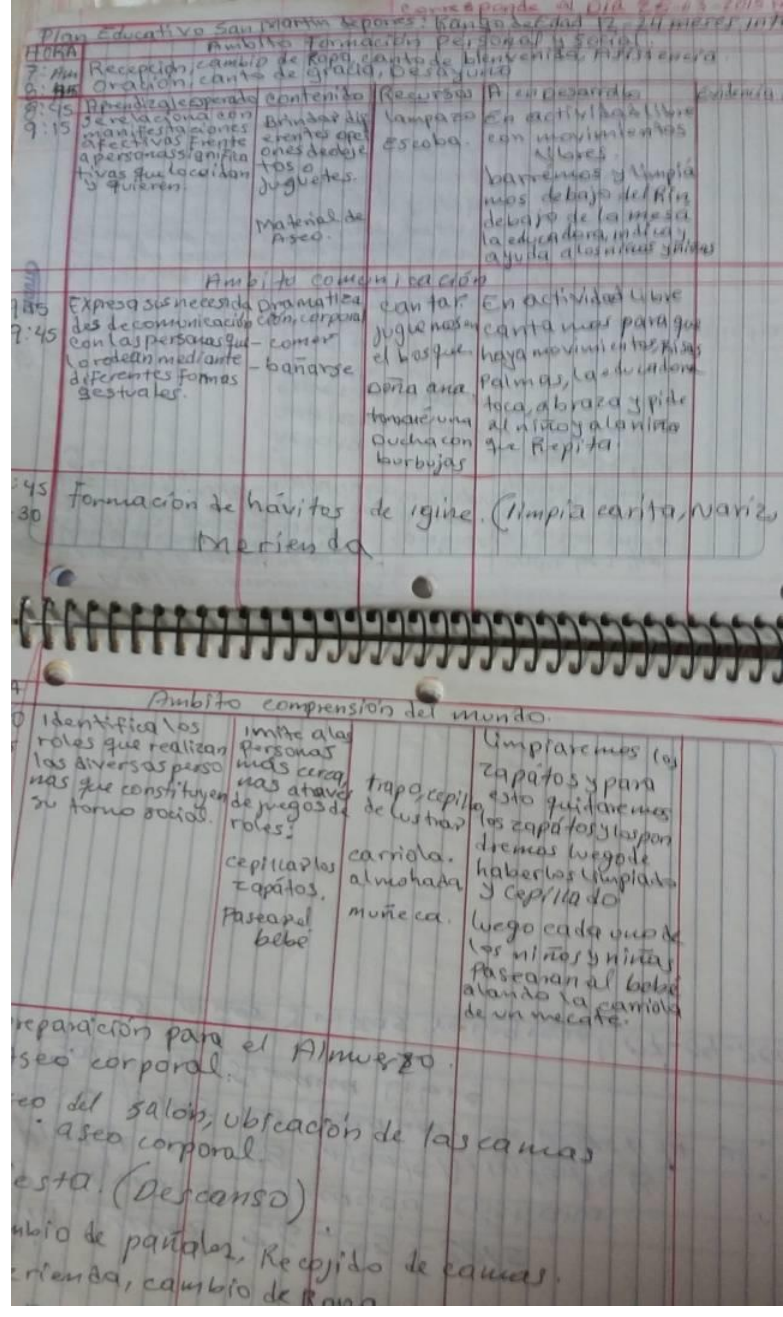

Figura 5. Planificación antes de capacitación

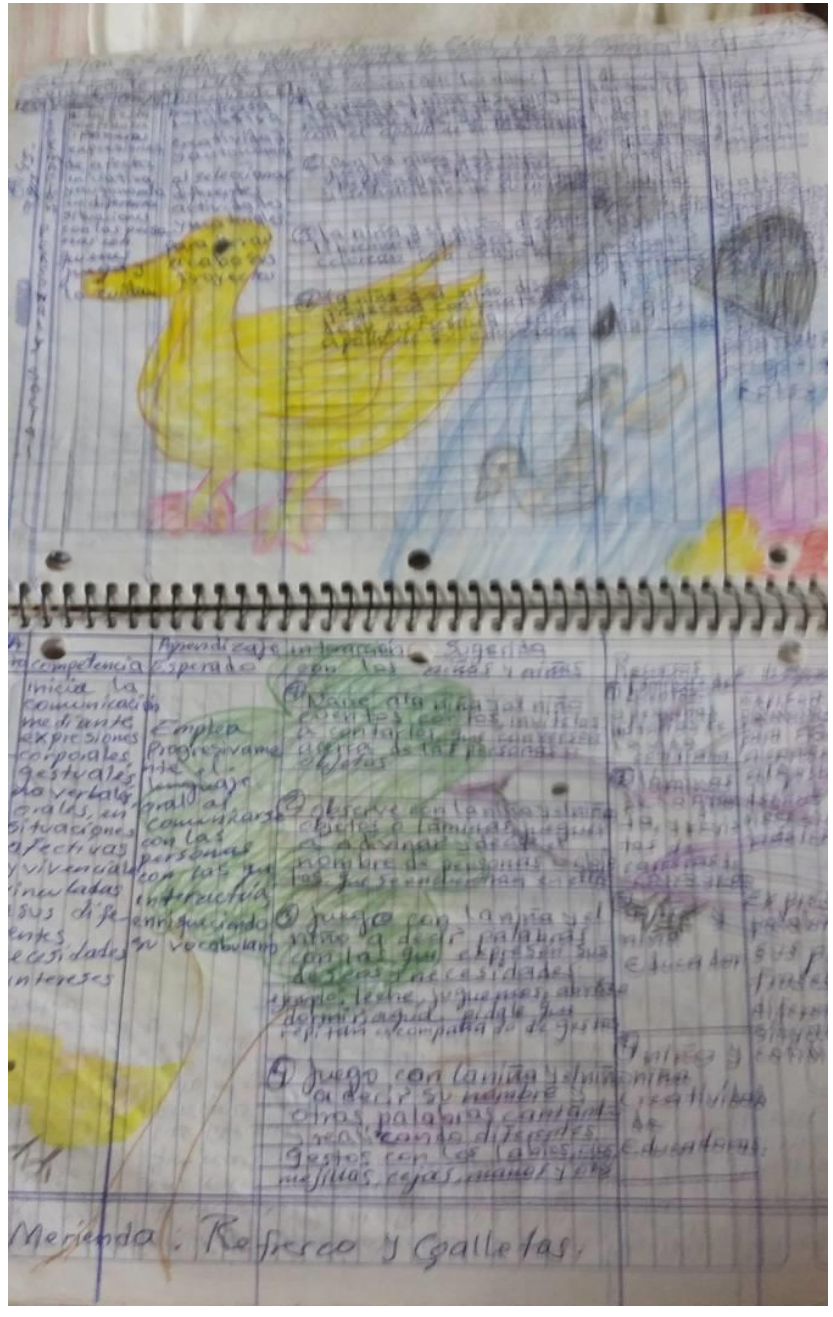

Figura 6. Planificación después de capacitación 
Afirmó, actualmente quien revisa los planes es el nuevo director académico, pero les revisa poco, porque son los niveles a los que dedica menor tiempo, ya que se dedica más a primaria y secundaria. Se hace un poco de acompañamientos en los Infantes pero es bien limitado, porque el Ministerio de la Familia no hace ese proceso, "nosotras nos indagamos con otra directora y educadoras de otro centro" expresó la directora.

Todos estos datos confirman la hipótesis de acción, ya que la intervención realizada produjo los cambios esperados, aunque se observó la presencia de algunas de las dificultades encontradas al inicio.

\section{DISCUSIÓN DE RESULTADOS}

Según la Política de Primera Infancia, en su apartado "Calidad de los programas", menciona que todos los programas y centros educativos, deberán disponer de personal especializado para atender en sus diferentes campos a la niñez. Así mismo, una de las líneas de acción sobre Centros de Desarrollo de Primera Infancia, implica capacitar a las educadoras, para que dominen los marcos conceptuales y metodologías pertinentes para la primera infancia. Sin embargo este estudio refleja que existe necesidad de capacitación y formación permanente de las personas que brindan atención educativa a los niños menores de tres años.

La formación de las maestras de educación inicial, suele ser un reto muy necesario, esto será posible mediante la aplicación rigurosa de las líneas de acción, ya propuestas por las autoridades en las políticas y planes estratégicos para mejorar la calidad de la educación, que involucren a todos los formadores de educación inicial, para mejorar la calidad que se pretende alcanzar.

Por otra parte, esta investigación refleja que aunque las educadoras realizan planificación de las experiencias de aprendizaje, y éstas eventualmente son oportunas de acuerdo a la edad de los niños, se observó deficiencias en el planteamiento y aplicación de estrategias, aun cuando el principio de integralidad de la Política de Primera Infancia de nuestro país hace énfasis en que la atención educativa que ha de brindarse a los niños menores de tres años, debe responder al desarrollo de las capacidades y potencialidades, fundamentándose en la necesidad de aplicar estrategias que garanticen el cumplimiento de los derechos de los niños, y atiendan sus necesidades, características e intereses.

En la entrevista realizada al Técnico del Ministerio de la Familia, este hace alusión al principio de libertad, refiriéndose a que no se promueve la planificación diaria en el ciclo 1 de educación inicial, porque la planificación debe ser flexible, y que respetando el principio de libertad las actividades se 
cambian, por otra el niño debe mostrar interés. Respecto a esto cabe mencionar por ejemplo, las escuelas de María Montessori, quienes permiten al niño dentro de su libertad, elegir libremente, pero al mismo tiempo se proponen situaciones que lo invitan a decidir y aceptar límites de su propia libertad.

Por otra parte, es de suma importancia reconocer que el trabajo educativo de las docentes, su organización responde a las orientaciones que les han brindado, y para mejorar su práctica educativa es necesario que las autoridades les brinden mayores opciones de planificación, tomando en cuenta los distintos niveles de planificación, para poderlos efectuar y articular.

También, se debe considerar la intención de cambio y mejora de su labor, aunque las orientaciones generales que les brindan no hayan permitido realizar otros cambios oportunos, se sugirió la modificación del plan para poder plantear adecuadamente sus objetivos, estrategias y formas de evaluación.

Cabe mencionar que una de las limitantes para este estudio, es que se solicitó a la directora autorizara que los niños se retiraran un poco antes los días en que se capacitarían a las educadoras, inicialmente aceptó, pero el primer día expresó que no era posible, y que los talleres debían desarrollarse durante los niños descansaran reduciendo el tiempo para realizar las capacitaciones.

\section{CONCLUSIONES}

El estudio refleja la necesidad de capacitación y formación permanente de las personas que brindan atención educativa a los niños menores de tres años, Se brindan algunas sugerencias de estrategias pertinentes para aplicarla a niños de esas edades, Algunas educadoras mostraron mayor interés al realizar su planificación, esto se reflejó en la estética del plan, la redacción paso a paso de una o dos estrategias propuestas y expresiones manifestadas durante la presentación del informe.

\section{RECOMENDACIONES}

Las principales recomendaciones están dirigidas al Técnico del Ministerio de la Familia: Orientar a las docentes la elaboración de planificación anual, mensual y semanal, como herramienta para facilitar la creación del plan diario; brindar acompañamiento pedagógico a las educadoras, dirigido a la preparación y planificación de diversas estrategias diarias a desarrollar con los niños, de forma práctica y sencilla. 
A la directora del Centro educativo: Proporcionar recursos didácticos y complementarios para la planificación de las docentes del ciclo 1 de educación inicial y así mismo, orientar nuevas y variadas estrategias de planificación de las clases.

A las educadoras: Retomar las orientaciones brindadas en los talleres, tomando en cuenta la importancia de describir las actividades y sus estrategias a realizar diariamente, en su formato de plan semanal; seleccionar diversas estrategias e incorporarlas a su planificación, para evitar repetir las mismas actividades de forma consecutiva, o improvisaciones, lo cual les permitirá preparar sus recursos con anticipación y considerar la opción de planificación semanal presentada, para mejorar el planteamiento de las estrategias a realizar.

A otros investigadores: Se sugiere a personas interesadas en esta área de investigación, dar continuidad a este estudio, valorando y la importancia del tema y la situación de la planificación didáctica en otros centros de atención a la niñez de 0-3 años.

\section{REFERENCIAS}

Gervilla, C. Á. 2006, Didáctica Básica de la Educación Infantil, Ediciones Narcea, España. Recuperado de:

https://books.google.com.ni/books?id=JH6P9MA8XXkC\&pg=PA25\&lpg=PA22\&dq=maria+mo ntessori+polarizaci\%C3\%B3n+de+la+atención

Manhey, M. (2012) Pedagogía de la Primera Infancia,) Módulo III, “Contextos que favorecen el aprendizaje de los niños y las niñas”: Planificación, tiempo, espacio, evaluación y ambiente humano.

Ministerio de la Familia, niñez y adolescencia de Nicaragua, Guía para la Aplicación del Currículo 0-3 años en Centro Infantil Comunitario y Centro de Desarrollo Infantil, Nicaragua, 2013.

Ministerio de la Familia, niñez y adolescencia de Nicaragua, Programa de Atención Educativa a la niñez de 0-3 años, Managua-Nicaragua, 2013.

Ministerio de la Familia, niñez y adolescencia de Nicaragua, Política Nacional de Primera Infancia, Managua Nicaragua, 2011. 